\title{
Study of Different Parameters on the Chassis Space Frame For the Sports Car by Using Fea
}

\author{
Nagarjuna Reddy.Y, Vijaya Kumar.S \\ (M. Tech Student, Dept of Mechanical Engineering, \\ K.S.R.M College of Engg, Kadapa, Andhra Pradesh, India) \\ (Assistant Professor, Dept of Mechanical Engineering, \\ K.S.R.M College of Engg, Kadapa, Andhra Pradesh, India)
}

\begin{abstract}
The concept of Finite Element Analysis of a chassis space frame has been highlighted in this project. The topic has constrained the study of the chassis space frame. Complex assemblies are to be avoided, for sports car. The model of the chassis space frame is built using CATIA V5 and then imported to ANSYS CLASSIC 11.0 to find its finite element module. To perform a torsion and Bending Test on the computational prototype chassis to determine its torsional stiffness. To incorporate a design improvement study and note the effects on the global torsional stiffness of the chassis. The stress ,strength and degrees of freedom of the chassis will be investigated. The body shape is fixed and therefore the overall external shape of the chassis must not be altered. The engine bay must remain as open as possible to allow a variety of engines to be fitted.
\end{abstract}

Keywords: FEA, Sports car chassis.

\subsection{Sports car chassis}

\section{Introduction}

Chassis frames can also be considered as the structures. A carefully weighed arrangement of material that is intended to resist loads is called as structures. Automotive chassis space frame is a skeleton material on which most of the mechanical parts that include the tires, brakes, engines, and etc. are bolted. The chassis usually includes longitudinal channels placed in a pair and multiple transverse cross members that intersect the channels. The space frame in a vehicle includes running gear and the basic structure such as the drive shaft, suspension, transmission and engine. Even the body of a vehicle is entirely supported by a chassis (to let the vehicle get completed). The space frames are basically manufactured with steel or aluminium. The increasing use of aluminium for manufacturing the space frames in present world is what can be observed.

\subsection{Different Types of Chassis Frames}

The different types of chassis that are available in the market are :

- Ladder chassis.

- Back bone chassis.

- Monocoque chassis.

\subsubsection{Ladder Chassis}

This is the earliest kind of chassis. It looks like a ladder, so for that sake it is called a ladder chassis. The construction of this chassis is two longitudinal rail interconnected by many lateral braces. The rigidity to the structure is provided by the cross members and lateral. Most SUV's are still built up on them, though these types of space frames are not much used in the present day.

\subsubsection{Back Bone Chassis}

It is simple in structure with a study tubular backbone which joins the front and rear axle and is responsible for most of the mechanical strength of the frame work. At the end of the chassis, the suspension and the drive train are connected. From inside, it resembles the drive shaft tunnel or more conventional front engine vehicles, but the difference is that it was closed in the bottom surface to provide a true tubular section. Still when the torsional stiffness of a chassis is derived from one large central tube running the length of the car, the resistance to twist depends mostly on the cross sectional area of that tube, and it is clearly possible from that cross section to be much larger than that of a typical drive shaft tunnel.

\subsubsection{Monocoque chassis}

This type of chassis is used by most of the modern vehicles, as it is a single piece frame work which gives the perfect shape to the car. It is very much different from the ladder and the back bone type chassis. 
When several pieces are welded together, a one-piece chassis is built up. Unlike the above two types of chassis, it is incorporated with the body in a single piece, where as the former only supports the stress members.

\subsection{Purpose of Chassis Frames in a Vehicle}

i. The main purpose of the chassis frame is that, it carries all the mechanical parts of a vehicle like tires, engine, axle assemblies, steering and brakes and all these mechanical parts are bolted to this skeletal frame.

ii. Under any conditions, the strength, and stability is provided to the vehicle by these chassis frames.

iii. One of the basic purposes of the chassis frame is to rigidly connect the front and rear suspension while providing attachment points for different systems of a car.

iv. Throughout the automobile, low levels of noise, harshness and vibrations are ensured by the frames.

v. The weight efficiency of the vehicle is improved by the space frame chassis only.

vi. A proper and structured built frame improves the crash worthiness and also the safety of a passenger while driving.

\section{Idealization of a Chassis Frame}

The main aim of this project is to design a chassis space frame and determine its stresses, torsional stiffness, bending stiffness, cross sectional area and the moment of inertia of the frame by using finite element analysis and thus the final procedure of modal analysis follows at the end. A specific load is to be applied at the centre where the entire weight of the chassis space frame can be suspended.

Firstly and very firmly a space frame from ALMA building was selected. The model of the space frame is a Class 1 type racing vehicle. The weight, height and the dimensions of the space frame were measured using ruler, weighting gauges. These instruments were used from the metrology lab. It is a steel space frame. The overall length of the chassis is $2650 \mathrm{~mm}$, the wheelbase is $2000 \mathrm{~mm}$ and its weight is 35 kilograms.

The measurements obtained were implemented in the computer using the software CATIA V5. After the design was prepared it was imported into ANSYS Classic 11.0 for analyzing. Static analysis and modal analysis was done to find out the stresses of the space frame and all the 6 Degrees of freedom.

The model was first developed using CATIA V5 software. The procedure in brief for this is as follows: -Firstly 4 points were located on a surface and then denoted as point 1,2 3 and point 4 . Next 4 lines were allowed to join these 4 points and then sweep was used to sweep all the 4 joined points and in this way the entire procedure was carried out and finally the chassis space frame was designed using this software of Catia V5.

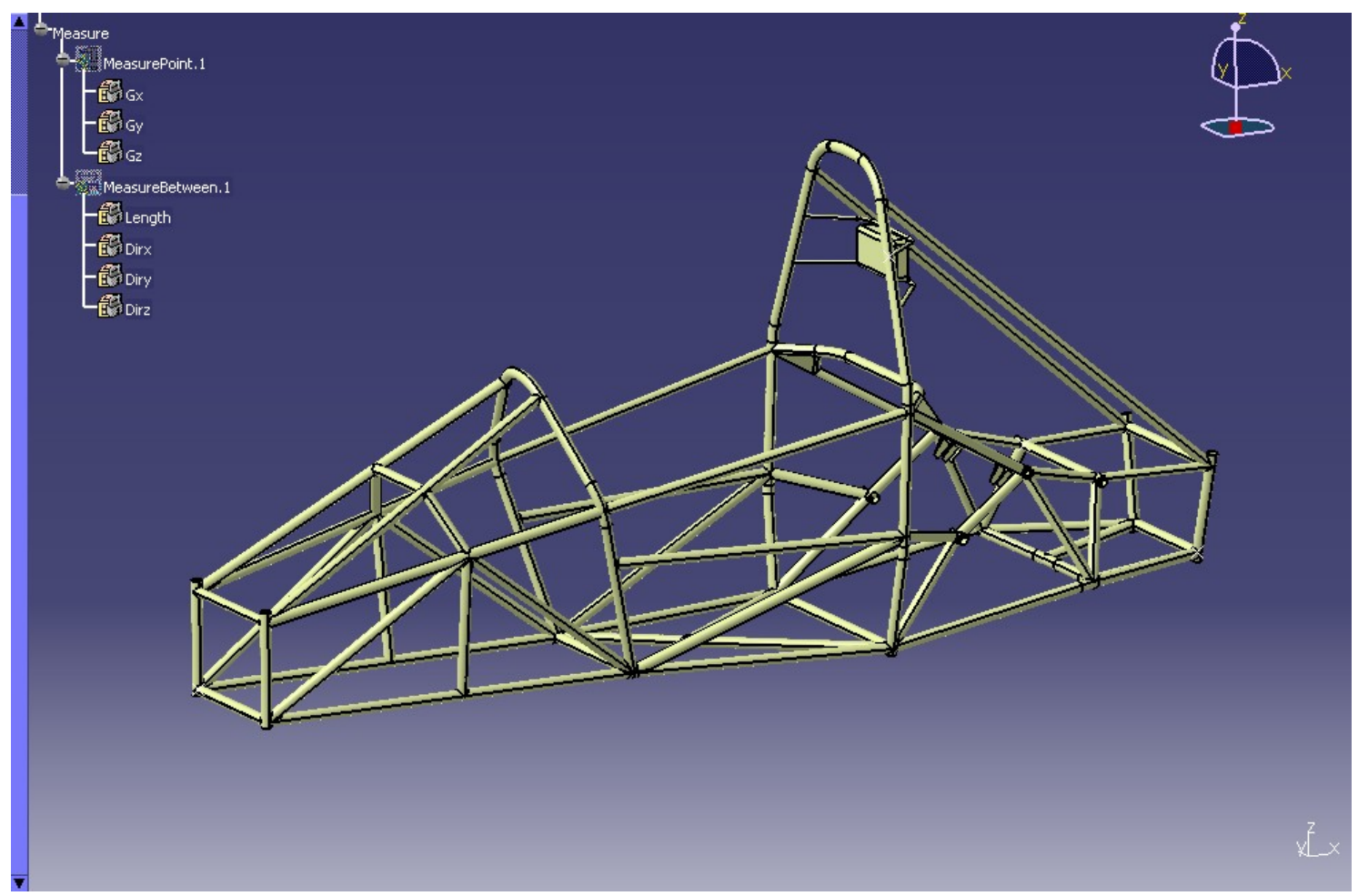

Fig 1 Chassis Frame Designed in CATIA

This is the design of the class 1 model chassis space frame designed using Catia V5, and after this, the design was imported to Ansys Classic 11.0 and its procedure is mention below. 


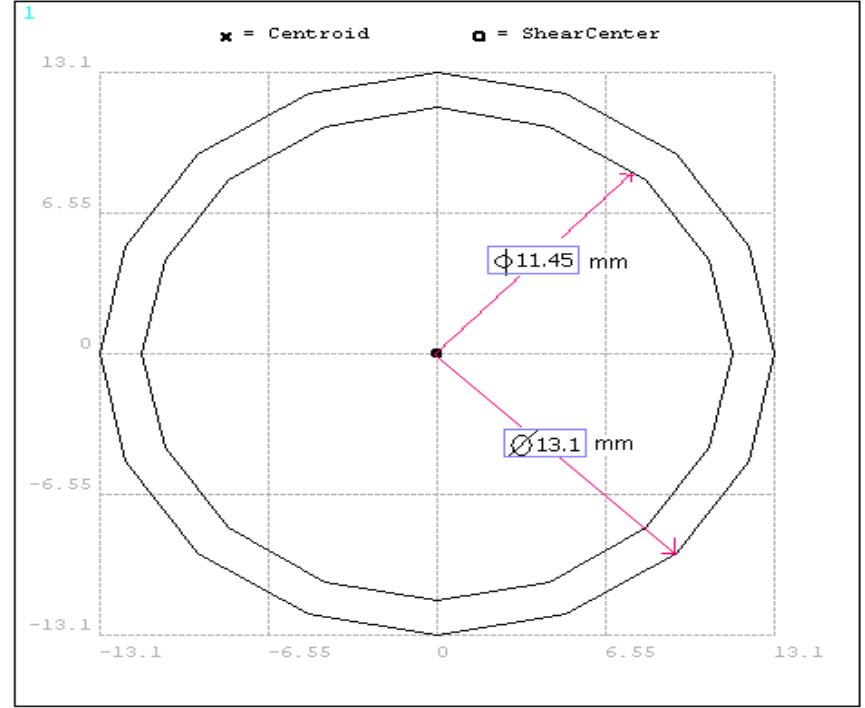

DATA SUMMARY

Area

$=127.159$

$\stackrel{I_{Y Y}}{=} 9611$

IYZ

$\mathrm{Izz}$

Warping Constant

$=0$

Torsion Constant

$=19222$

Centroid Y

Centroid $z$

$=-.151 \mathrm{E}-14$

Shear Center

$=.595 \mathrm{E}-16$

Shear Center 2

$=.129 \mathrm{E}-14$

Shear Corr. $\mathrm{YY}$

$=.503247$

Shear Corr. Y

$=-.405 \mathrm{E}-13$

Shear Corr. $\mathrm{ZZ}$

Tubular cross section properties

Fig -2 Tubular Cross Section Properties

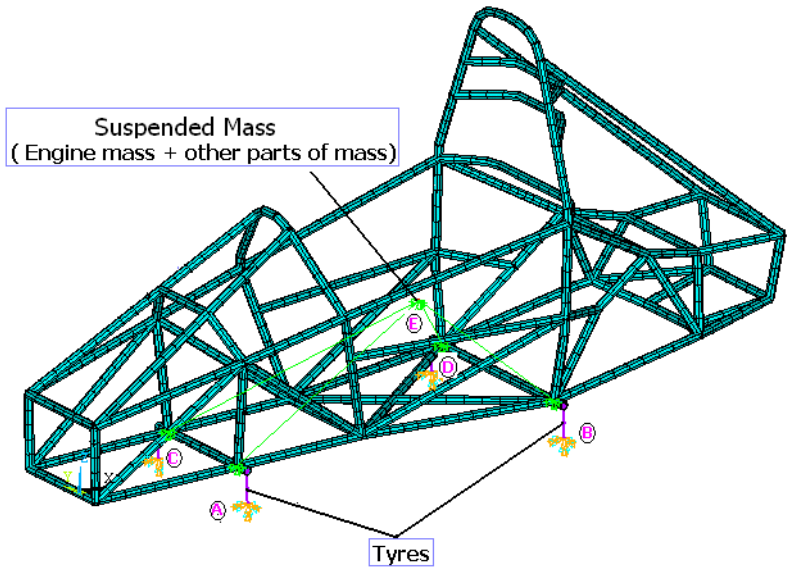

Fig 3 Finite Element Model of a Chassis Frame

\subsection{Race Car Chassis Frame Model Description}

Race car chassis frame model is selected for analysis purpose. The overall length of the chassis is $2650 \mathrm{~mm}$, the wheelbase is $2000 \mathrm{~mm}$ and its weight is 35 kilograms.

\subsubsection{Assumptions}

Any problem becomes complex if the real situation are considered. It becomes very difficult to analyze the problem with such complexities. In order to simplify the problem, some assumptions are made. In the present analysis the following assumptions are made

- The tyres are considered as linear springs.

- The mass of the engine gearbox and the other components are lumped at exactly placed at centre of gravity location of nodes.

\subsubsection{Ansys Model for Race Car Chassis Frame}

The ultimate purpose of a finite element analysis is to re-create mathematically the behaviour of an actual engineering system. Model comprises all the nodes, elements, material properties, real constants, boundary conditions and other features that are used to represent the physical system.

The chassis discretized by taking into account 3D- space frame idealization and it was done by using 2node tapered beam element with each node having 6 DOF with tube a thickness of $1.65 \mathrm{~mm}$ and combination element is used for suspensions and tyres. Structural mass element for various components like engine, gearbox, 
and total sheet metal body have been used lumped at the appropriate node locations. Total number of elements and nodes of the model are 900 and 850 respectively.

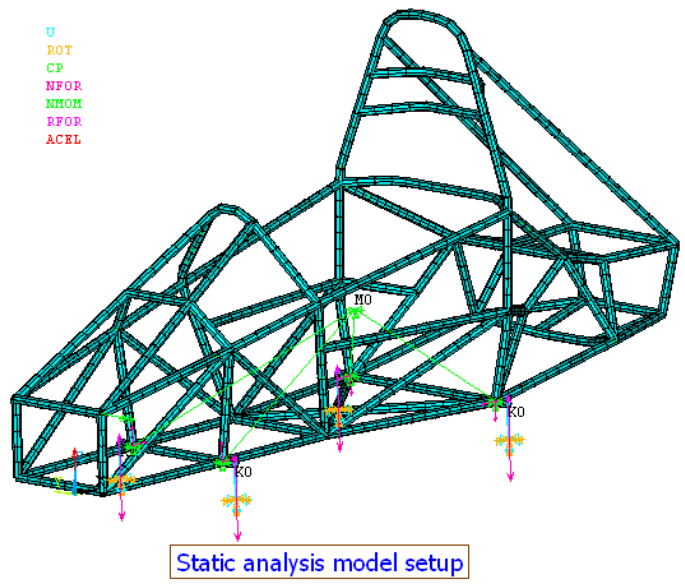

Fig 4 Static Analysis Setup Model

\subsubsection{Boundary Condition}

Following displacement constraints are applied in the present modelling.

- Three times of gravity load is applied to the weight of the chassis body (3G beaming load), tyre contact patch location (i.e. A, B, C and E) nodes were constrained all degree of freedoms to carry out the applications of static analysis.

- In modal analysis the bottom points of tyres are fully arrested in all degrees of freedom.

\subsection{Static Analysis}

Static analysis was carried out for entire space frame model which was made of steel tubular section. Main objective of the static analysis is to find the bending stress and shear stress due to $3 \mathrm{G}$ beaming load condition. In analysis finding out the structures, whatever stresses are coming below yield point of the material.

\subsubsection{Procedure for Static Analysis}

- The model using linear elements by using tapered beam44, combination14, structural mass21 was created and coupled with mass to the structure by couple-couple equations.

- The material properties of linear isotropic such as young's modulus $=206900 \mathrm{MPa}$ and density of steel and poisons ratio $=0.27$ have been defined.

- The lumped mass like engine, gear box, propeller shaft, including the lumped mass, passengers and driver's mass placed on the chassis at appropriate centre of gravity nodes locations.

- The nodal degrees of freedom at the bottom nodes of the tyre in all directions have been arrested.

- Enter the ANSYS solution Pre-processor in which analysis type is modal analysis, and by selecting static method, solution method is continued by using linear static method.

- The problem is solved by using current LS command from the solution menu bar

\subsection{Modal Analysis}

Modal analysis is used to determine the vibration characteristics (natural frequencies and mode shapes) of a structure or a machine component while it is being designed.

- The main aim of modal analysis is to obtain the Eigen frequencies, eigenvectors and different mode shapes of the model at different frequencies.

- Block Lanczo's method was used for modal analysis with the help of ANSYS. Block Lanczo's method is used to find out the closed spaced eigenvectors of large symmetric matrix. This method was used for trigonalization only.

- The frequency response function is displayed between any two-measurement points on the vehicle independent of the exciter locations chosen for the actual test data collection.

- Display the response the vehicle at any single point to any type of specified input force at any other point.

- Display in animated form, the natural mode shapes of the vehicle.

\subsubsection{Procedure for Modal Analysis}

- A model using linear elements is created because only linear behaviour is valid in the modal analysis. 
- The material property of linear isotropic such as young's modulus $=206900 \mathrm{MPa}$ and density are defined.

- The lumped mass like engine, gear box, propeller shaft, including above lumped mass, passengers and drivers mass placed on the chassis at appropriate nodes locations.

- The nodal degrees of freedom at the bottom nodes of the tyre in all directions are arrested.

- $\quad$ Enter the ANSYS solution processor in which analysis type is taking as modal analysis, and by taking mode extraction method, by defying number of modes to be extracted.

- Solution method is chosen as block Lanczo's method.

- The problem is solved using current LS command from the solution menu bar.

\section{Results and Discussions}

Results are more important for any problem. In any analyses, the results that are efficient, useful and favourable are accepted. These should be practically applied to design or modify the model. An engineer fetches a lot with efficient and favourable results and will help him to apply his innovative ideas in obtaining a model which is much more efficient. Results should be consistent and assist the designer to design the vehicle to satisfy customer needs, safety, comfort driving, service and economic conditions.

\subsection{Task}

- To develop the finite element model of race car chassis, to predict the static and dynamic behaviour of vehicle using static modal analysis.

- $\quad$ All these analysis are carried out by using ANSYS CLASSIC 11.0.

- The race car chassis has been idealized using a finite element model as shown in Figures above.

- The model takes into account the thinned rectangular, tubular sectional members of chassis frames are modelled as taparedbeam 44 element, suspension and tyres are modelled as combination 14 element.

- Structural mass element for various components like engine, gearbox and body of frame lumped at the appropriate node locations.

\subsection{Centre of Gravity}

$$
\text { Centre of mass location } \begin{aligned}
X & =1179.2 \quad \mathrm{~mm} \\
Y & =-0.34809 \mathrm{~mm} \\
Z & =323.89 \quad \mathrm{~mm}
\end{aligned}
$$

Moment of inertia about centre of mass $=$

$$
\begin{aligned}
& \mathrm{Ixx}=3876.0 \quad \mathrm{~mm}^{\wedge} 4 \\
& \mathrm{Iyy}=0.1848 \mathrm{e}+5 \mathrm{~mm}^{\wedge} 4 \\
& \text { Izz }=0.1768 \mathrm{e}+5 \mathrm{~mm}^{\wedge} 4 \\
& \mathrm{Ixy}=-4.664 \quad \mathrm{~mm}^{\wedge} 4 \\
& \text { Iyz }=3.594 \quad \mathrm{~mm}^{\wedge} 4 \\
& \text { Izx }=-1066 \quad \mathrm{~mm}^{\wedge} 4
\end{aligned}
$$

Stiffness calculation race car chassis frame.

For stiffness calculation we have applied unit load (i.e. $1 \mathrm{~N}$ force) on the chassis, due to this load maximum deformation on chassis is $0.633 \mathrm{e}-3 \mathrm{~mm}$ obtained.

1.8.1 Structural Stiffness of Member Formula

$$
[\mathrm{K}] *[\mathrm{X}]=[\mathrm{F}]
$$

Where $\mathrm{K}$ is called the stiffness

matrix, $\mathrm{X}$ is called the displacement matrix

and $\mathrm{F}$ is the load matrix

so,

$\mathrm{K}=\mathrm{F} / \mathrm{X}=1 / 0.633 \mathrm{e}-3$

$=1579.03 \mathrm{~N} / \mathrm{mm}$

Chassis stiffness is $1579.03 \mathrm{~N} / \mathrm{mm}$

\subsection{Results Obtained from Static Analysis}

From Static analysis it has been observed that maximum displacement of chassis is $0.043 \mathrm{~mm}$ from fig 6 From figure 7 to 10 indicating that maximum shear force diagram, bending moment diagram, bending stress plot and stress plot. Shear force detects the distribution of positive and negative forces that are being distributed among the structure whereas the prediction of stress distribution is done through bending moment diagram. Maximum stress was observed from fig 9 is $1.74 \mathrm{MPa}$, this stress value well below the yield point of the steel 
material (steel young's modulus $-206900 \mathrm{MPa}$, Poisson ratio -0.27 and yield point is $-240 \mathrm{MPa}$ ).Under the beaming loading condition structure is safe.
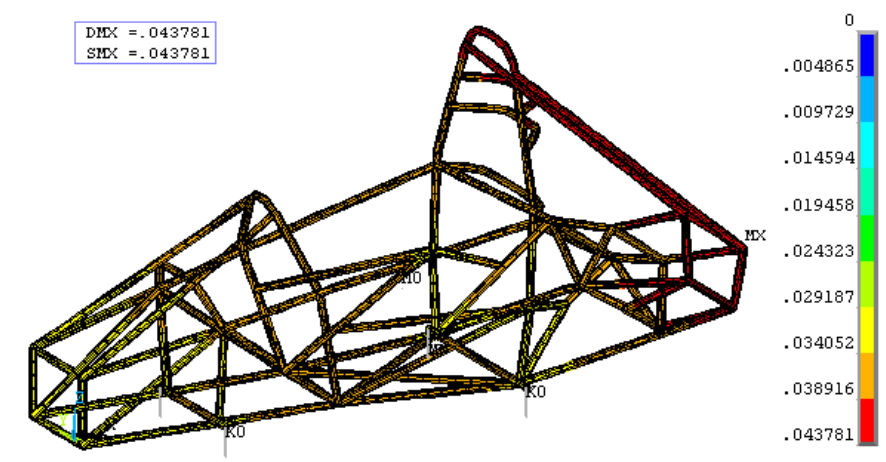

Displacement plot under the 3G beam loading condition

Fig 0 Displacement Plot under the 3G Beam Loading Condition

From the above design, the maximum displacement of the chassis is measured to be as $0.043 \mathrm{~mm}$

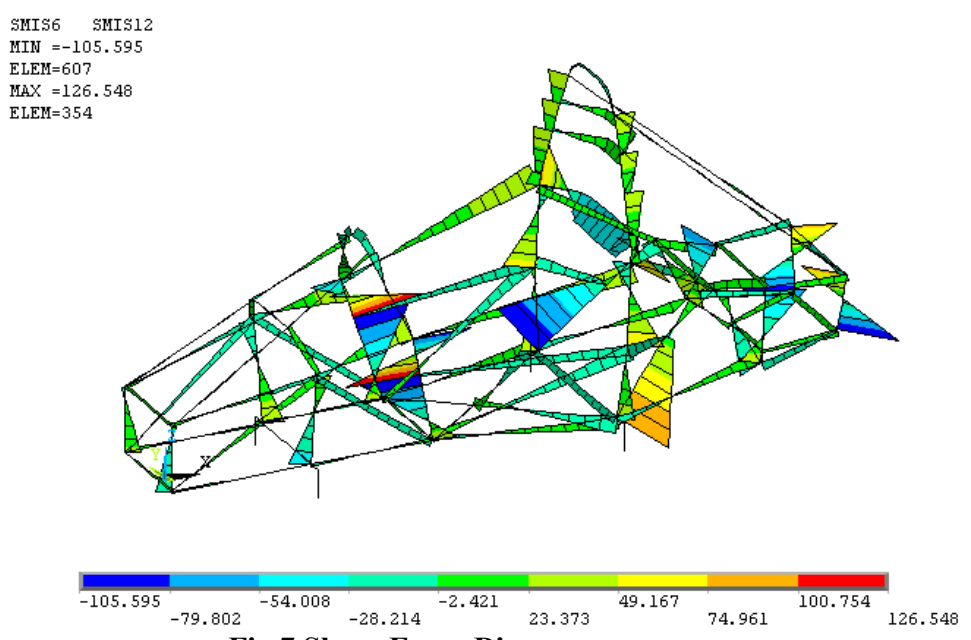

Fig 7 Shear Force Diagram

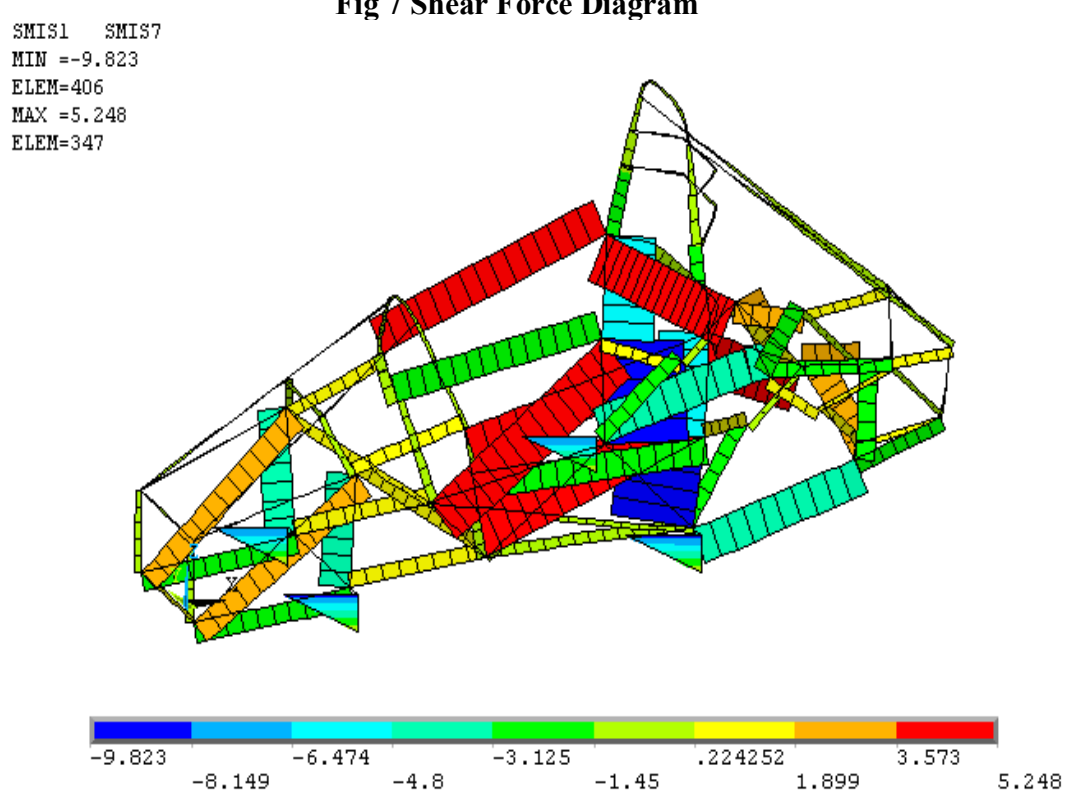

Fig 8 Bending Moment Diagram 

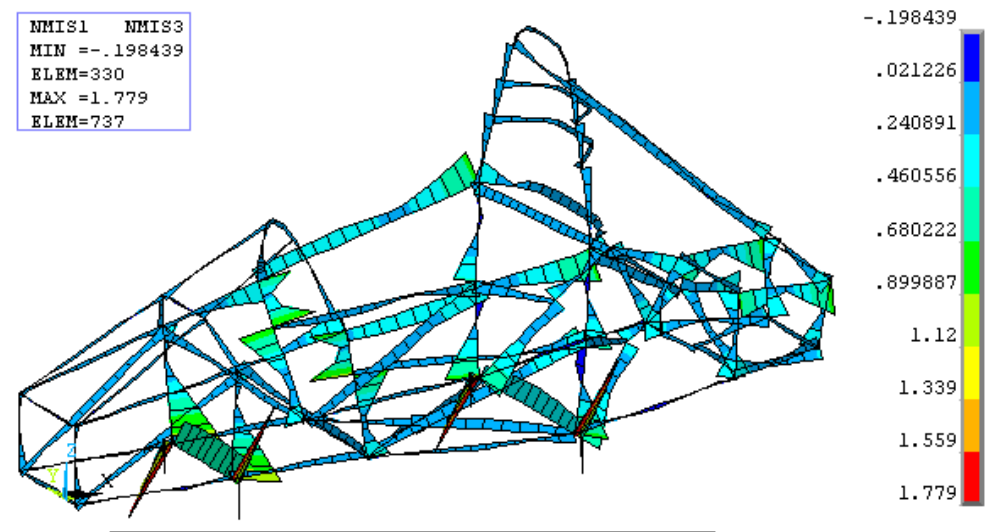

Bending stress under the load of $3 \mathrm{G}$ beam

Fig 9 Bending Stress Plot under Load of 3G Beam

The maximum bending stress under $3 \mathrm{G}$ beam loading is $1.779 \mathrm{~mm}$ at element 737 and the minimum bending stress is -0.198439 at element 330 .

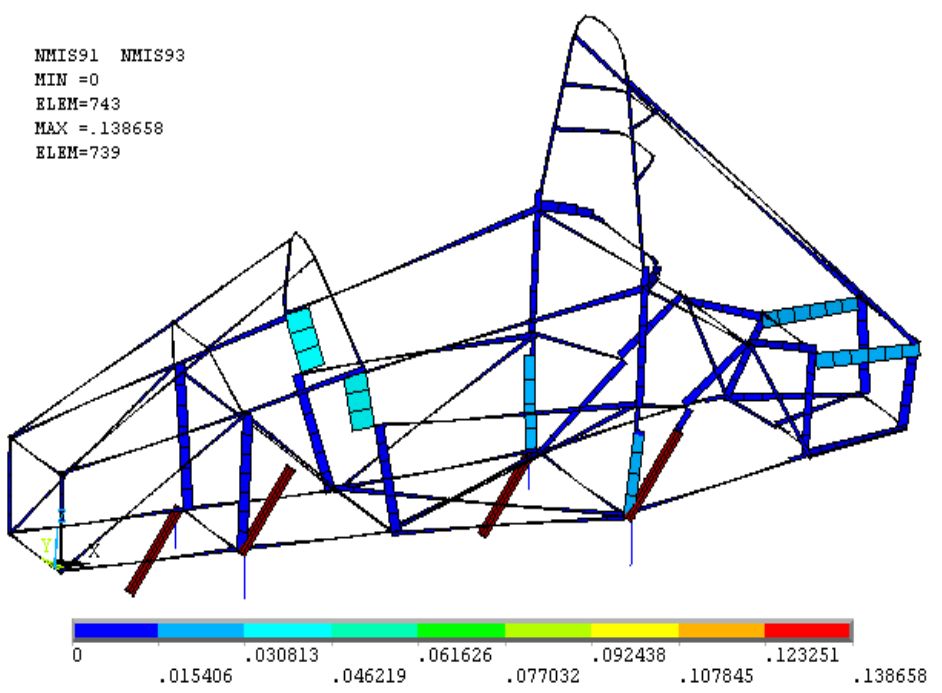

Fig 10 Shear Stress Plot under Load of 3G Beam

The maximum shear stress of the chassis space frame under $3 \mathrm{G}$ beam loading is $0.138658 \mathrm{~mm}$ at element 739 and the minimum is $0 \mathrm{~mm}$ at element 743 .

\subsection{Results Obtained From Modal Analysis}

The modal analysis is the sequential procedure applied to obtain the modal frequencies and mode shapes of the model.

- Block Lanczo's method was applied for obtaining the results.

- This method is very efficient (fast \& accurate) and also gives the closed spaced Eigen values for the analysis of large structures as it occupies less memory of the computer than any other methods.

- But the initial frequencies are much important, as this effect on the system behavior significantly. Thus the chassis model was analyzed for 1 to 6 mode shapes, and the first significant natural frequencies were obtained.

- The list of the natural frequencies and modes shapes of the model are given in the Table below as these mode shapes indicate, the physical behavior at each frequency.

\subsubsection{Mode Frequencies}

\begin{tabular}{|l|l|l|}
\hline Serial no. & Mode & Frequencies (HZ) \\
\hline 1. & Twist Mode & $42 \mathrm{~Hz}$ \\
\hline 2. & Bounce Mode & $48 \mathrm{~Hz}$ \\
\hline 3. & Pitch mode & $76 \mathrm{~Hz}$ \\
\hline 4. & Roll Mode & $73.6 \mathrm{~Hz}$ \\
\hline
\end{tabular}



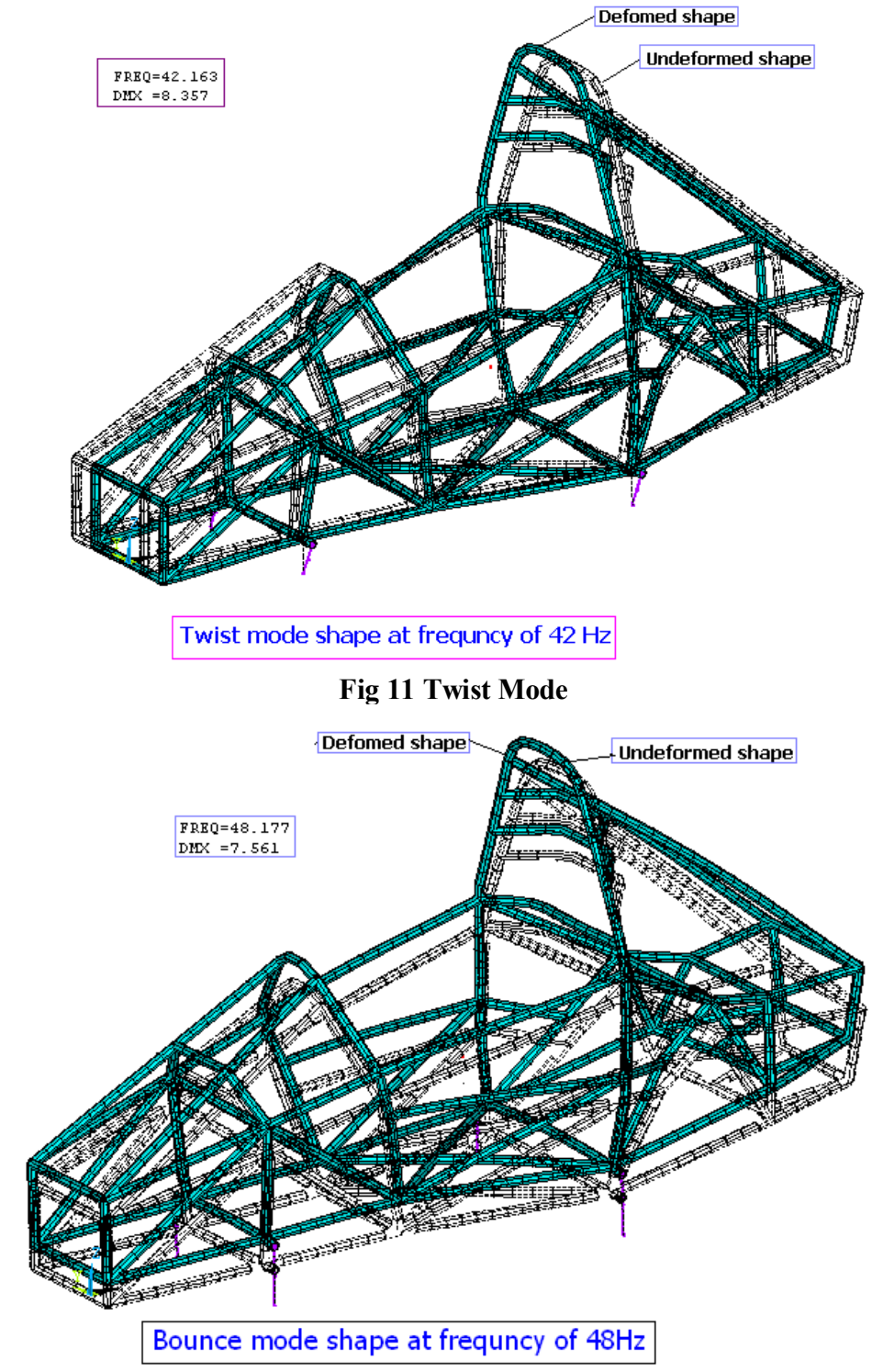

Fig 12 Bounce Mode

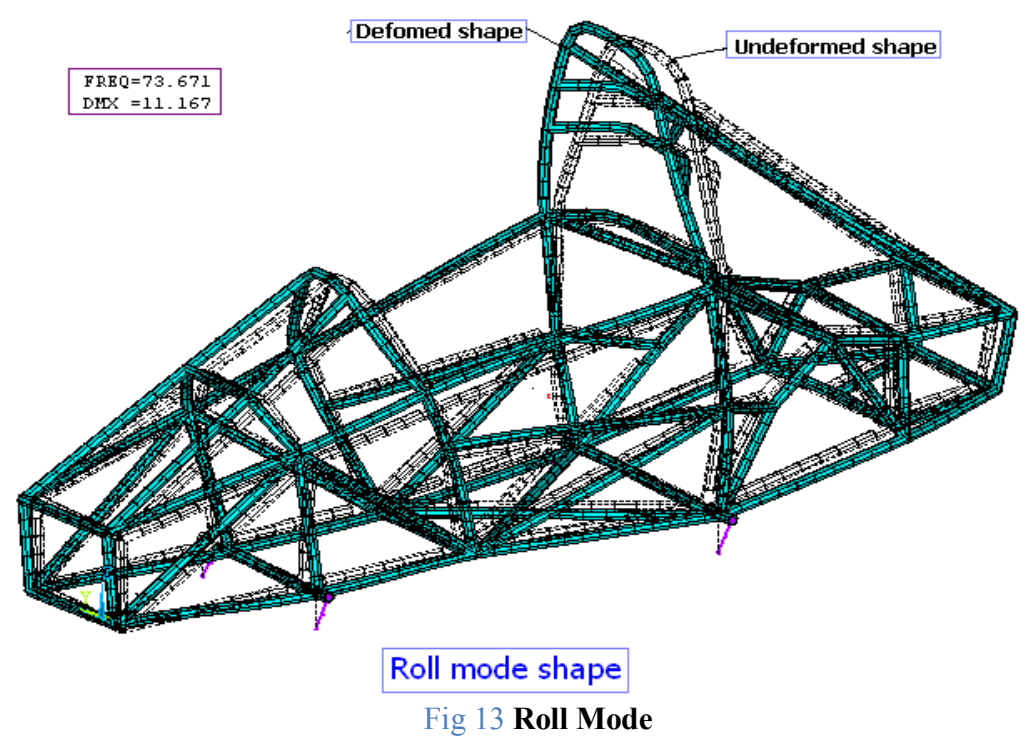




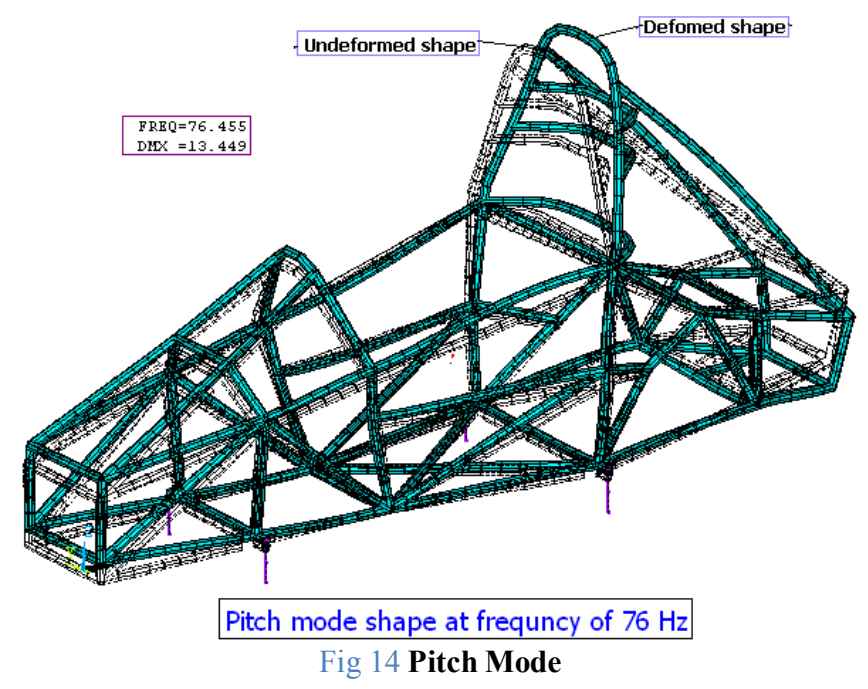

\section{Conclusion}

We have studied about the design and analysis of a chassis space frame and learnt about the analyzation processes like the static analysis and modal analysis in ANSYS classic, in determining the finite element module of a chassis space frame (CLASS 1 MODEL). Though the time available to deal with this project is limited, it didn't reflect in my research.Various topics and projects related to the chassis space frames like the static analysis, dynamic behaviour of engine chassis, FEA of chassis frame and the Eigen value analysis has been very successfully described and discussed in the literature review.The design which was done in CATIA V5 and then imported to ANSYS classic 11.0 to find the finite element analysis and its degrees of freedom has been made from the studies. Thus I would conclude by observing the above results and analyzation that the chassis space frame structure depend on the stiffness and stresses in that particular frame. The stresses obtained above are the well deserved in order to manufacture a chassis space frame as the stresses are very much lower as compared to that of the yield point of the material.

\section{References}

[1]. Aird, F. (2008). THE RACE CAR CHASSIS. New york: PENGUIN GROUP.

[2]. Ali, R. H. (n.d.). "The application of finite element techniques to the analysis of an automobile structure ", .

[3]. Balamurugan, V. (n.d.). "Dynamic analysis of a military tracked vehicle",.

[4]. Blair. (2002). Automotive (Vol. bsbcsb). London: sbvdbs.

[5]. C.S Manohar, S. V. (n.d.). "Finite element analysis of vehicle structure interaction during launching of remotely piloted air-vehicles".

[6]. C.Sujatha, V. a. (n.d.). "Bus vibration study-finite element modeling and determination of the Eigenpairs".

[7]. Chen., Y. T. (n.d.). "The design of an active suspension force controller using genetic algorithems with maximum stroke constraints",

[8]. Cook, R., Malkus, D., \& Plesha, M. (n.d.). "Concepts and applications of finite element analysis". 